\title{
Wie funktioniert eigentlich eine optimale Krisenprävention? Maßnahmen gegen den Super-Gau
}

Fast genau ein Jahr ist es nun her: Anfang Mai letzten Jahres erschreckten zunächst Meldungen in den regionalen und später auch überregionalen Medien über mehrere Fälle von Salmonelleninfektionen in einem großen deutschen Klinikum nicht nur die breite Öffentlichkeit. Hätte die Klinikleitung des betroffenen Krankenhauses damals richtig reagiert, hätte sich die Lage viel weniger zugespitzt. Doch welche Kommunikationsstrategie ist im Krisenfall angezeigt? Das muss sicherlich für jeden Fall individuell entschieden werden - andererseits gibt es einige klare Regeln, an die man sich halten kann. Das wichtigste ist dabei eine klare Kommunikation mit der Öffentlichkeit und relevanten Bezugsgruppen, denn dies schafft Vertrauen und signalisiert Kompetenz. Dann lassen sich Krisen auch meistern.

Die Salmonellenepidemie in einem hessischen Klinikum, die im letzten Jahr rund drei Monate die Medien mit bestimmte (vgl. Kasten), ist heute ein Paradebeispiel dafür, was im Verlauf einer Krise alles schief gehen kann. Fehlende Präventionsmaßnahmen, das Leugnen der Krise in der Akutsituation und eine überstürzte Kommunikation ohne Absprachen waren der Grund, warum das betroffene Klinikum weit über sein regionales Einzugsgebiet hinaus, zum Inbegriff eines Krankenhauses wurde, in dem man lieber nicht behandelt werden würde. Allerdings hätte die Situation auch viel glimpflicher ablaufen und der Schaden minimiert werden können. Wie? Mit einer zuverlässigen und umfassenden Krisenprävention.

Sicherlich hat jede Krise viele Grundlagen - drei Bereiche sind jedoch dabei entscheidend:

- die Prophylaxe durch optimierte Abläufe (wenn immer möglich)

- die Kommunikation mit der Öffentlichkeit

- die Kommunikation mit den relevanten Bezugsgruppen, den sogenannten Stakeholdern (z.B. Gesundheitsamt, das Robert-Koch-Institut, der Bürgermeister, Patientenverbände, die Verbraucherzentrale und viele mehr).

\section{Lassen Sie es erst gar nicht zur Krise kommen!}

Viele Krisen sind, ein gewisses Maß an Prävention vorausgesetzt, vermeidbar.
Dies gilt gerade auch für Ausbrüche von Infektionskrankheiten wie den Salmonelleninfektionen in unserem Beispiel. So könnten bereits im Vorfeld Sicherheitslücken im Arbeitsprozess der Küche, dem „Zentrum der Krise“, geschlossen werden, sei es die Kontrolle des Einkaufsprozesses (Lieferanten, Kühlkette) oder auch die Identifizierung risikorelevanter Lebensmittel (z.B. Fleischprodukte, Rohmilch, Eier, Tiefkühlprodukte) und sonstiger möglicher Infektionsquellen (z.B. Trinkwasser).

Auch dezidierte Handlungsanweisungen zum Umgang mit den risikobehafteten Produkten (Lagerung, Zubereitung), das Screening der Mitarbeiter sowie deren Schulung und Überwachung zählen in diesem Fall zu den wirkungsvollen Präventionsmaßnahmen. Ein Eingangsscreening der Patienten, die gleichermaßen als mögliche Infektionsträger infrage kommen, kann ebenfalls sinnvoll sein.

\section{Ziehen Sie im Ernstfall schnell die Konsequenzen}

Kommt es trotz solcher Maßnahmen zum Ernstfall, gilt es, schnell die richtigen Konsequenzen zu ziehen. Hätte die Klinik umgehend die Küche geschlossen und übergangsweise durch einen externen Anbieter ersetzt, die Patienten und Einweiser informiert sowie alle Mitarbeiter der relevanten Bereiche gescreent und später erneut kontrolliert, hätten die Ärzte die aufgetretenen Sal-
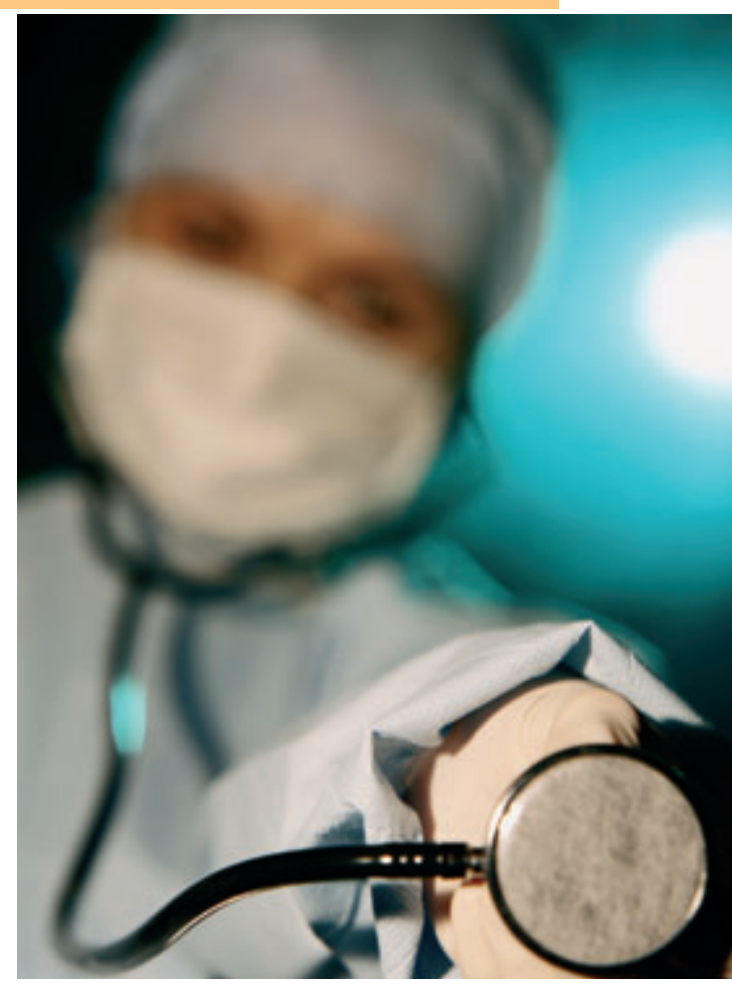

monelleninfektionen schnell in den Griff bekommen, und die zweite Infektionswelle wäre erst gar nicht aufgetreten. Die Krise wäre also erst gar nicht entstanden.

In unserem Beispiel blieb die Küche jedoch noch etwa zwei Wochen geöffnet, die zentralen Abläufe wurden nicht sofort hinterfragt, und die potenziellen Quellen wurden nicht schnellstmöglich eliminiert - mit schlimmen Folgen.

\section{Offene Kommunikation schafft Vertrauen}

Das zweite Standbein einer gelungenen Krisenprävention ist die Kommunikation mit der Öffentlichkeit, und die will gelernt sein. So sollte es nur einen zentralen Ansprechpartner für die Medien geben, der sich um einen engen und regelmäßigen Kontakt mit den Medienvertretern bemüht. Kann er dies Umsetzen, erhält er in möglichen Krisensituationen einen „Vertrauensbonus“. Ein mit der Klinik gut vertrauter Journalist wird zunächst mit „seinem“ Pressesprecher Kontakt aufnehmen und den Fall ansprechen, bevor er ungefilterte Informationen an die Öffentlichkeit bringt. Ideal ist darüber hinaus, wenn die Klinik eine Kommunikationsroutine etabliert hat, die dazu beiträgt, die Anforderungen an die Kommunikation in bestimmten Situationen schnell und sicher zu beurteilen. Wann zum Beispiel ist es sinnvoll, mit allen verfügbaren Informationen nach außen $\mathrm{zu}$ gehen, und in 
Wie die Salmonellen zum Krisenfall wurden

Anfang Mai 2007 gab es die ersten Meldungen über Fälle von Salmonelleninfektionen in einem großen deutschen Klinikum in der regionalen Presse und in der Frankfurter Rundschau. Die Klinikleitung wiegelte zunächst $a b$, der Direktor der medizinischen Klinik kommentierte „Grillsaison ist Salmonellensaison". Der Vorstandsvorsitzende des Hauses wiederum fuhr in den Urlaub, er war der Meinung: „Wir haben die Situation im Griff“.

\section{Absprachen unter den Verantwortlichen? - Fehlanzeige! \\ Am 10. Mai erklärte die Klinikleitung, die Epidemie sei unter Kontrolle - aber nur zwei Tage später berichten die Medien über eine „zweite Welle“ mit 94 Erkrankten und zwei Todesfällen. Am 20. Mai brachte Klaus-Die- ter Zastrow, Sprecher der Deutschen Gesell- schaft für Krankenhaushygiene, auch noch eine mögliche Sabotage mit ins Spiel, und der Vorstandsvorsitzende der Klinik geriet mit Fragen wie "Sollen wir jetzt nach Osama bin Laden suchen?", seinem Urlaub und seine zögernde Informationspolitik unter Druck. Der Sprecher des Klinikums wie- derum setzte auf „Daten, Zahlen und Fak- ten" und ignorierte damit mögliche Ängste der Bevölkerung. \\ Nach dem Ausschluss eines Sabotageakts spitzte sich am nächsten Tag das Vokabular weiter zu: Die Rede war jetzt von „tödlicher Schlamperei“, der ärztliche Direktor sprach von einem "Super-Gau“, der Bürgermeister von einer "schicksalhaften Heimsuchung“. Der Leiter des Gesundheitsamts sah die Lage}

welcher Form sollte dies geschehen? Generell ist anzuraten, immer die Position des Aufklärers einzunehmen und die Öffentlichkeit frühzeitig, umfassend und ehrlich zu informieren.

Gerade die sogenannte „Salamitaktik“, bei der Informationen nur auf Nachfrage - sprich „scheibchenweise“ - herausgegeben werden, kann Journalisten verärgern und $\mathrm{zu}$ einer intensiveren Recherche anderer Quellen verleiten. Die Öffentlichkeit reagiert auf ein solches Vorgehen ebenfalls empfindlich. Besser ist, man ist selbst eine verlässliche Informationsquelle.

\section{Vermitteln Sie Sicherheit und Souveränität}

Außerdem kann ein sogenanntes „Krisenhandbuch" bei dem oft schwierigen Umgang mit der richtigen Kommunikationsstrategie helfen. Hier können relevante Materialien, Kernbotschaften und Argumentationshilfen, zum Beispiel kurze, vorformulierte Statements zur Wiedergabe einer angemessenen, ersten öffentlichen Reaktion, sowie ein Katalog mit erneut „unter Kontrolle“. Patientenverbände und die Verbraucherzentrale bemängelten die langsame Reaktion des Krankenhauses auf das Auftreten der Infektionen. Auch die Politik mischte sich ein: Ein Vertreter der SPD sprach von einer „Geschichte aus dem Tollhaus" und hielt Management und Verantwortungsträger der Klinik für überfordert.

\section{Mit dem Fund von Legionellen ging es} „in die zweite Runde“

Anfang Juli wurden Legionellen in den Wasserleitungen des Klinikums gefunden. Sofort stieg das Interesse der Medien erneut, denn aufgrund der Salmonellenkrise war die Öffentlichkeit besonders wachsam und bereits für „Horrormeldungen“ aus der Klinik sensibilisiert. Zu diesem Zeitpunkt distanzierte sich das Kreisgesundheitsamt öffentlich von der Klinikleitung. Mitte Juli legte der Vorstandsvorsitzende sein Amt nieder. Der Ärztliche Direktor stritt in der Frankfurter Allgemeinen Zeitung (FAZ) sämtliche Versäumnisse ab und sprach von „unvorhergesehenen Ereignissen“. Die Verzögerungen bei der Meldung des Vorfalls wurden mit internen Kommunikationsproblemen begründet.

\section{Nur langsam beruhigte sich die Lage}

Ende Juli wurden die Ergebnisse der regelmäßig durchgeführten Salmonellenuntersuchung bei Mitarbeitern unter der Headline "Skandal-Serie“ publiziert. Erst langsam beruhigte sich das Interesse der Medien nach drei Monaten direktem Beschuss.

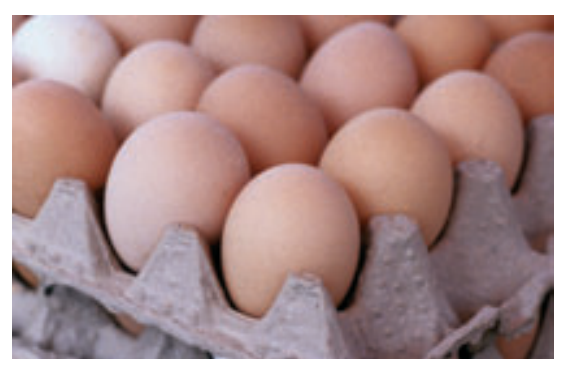

möglichen kritischen Fragen und Antworten im Rahmen eines Krisenhandbuchs als „erste Hilfe“ zusammengestellt sein. Denn ein nach außen geschlossenes Auftreten und fest definierte Sprachregelungen sind das A und O der Kommunikation. So erhält der Sprecher der Klinik Sicherheit und signalisiert Souveränität. In unserem Fall war die Klinik schlecht beraten, sich nicht durch einen Pressesprecher, sondern durch die medial nicht geschulte Klinikleitung nach Außen repräsentieren zu lassen. Diese spielte die Situation zunächst herunter, gab dann - obwohl dem nicht so war zweimal „Entwarnung“. Die Öffentlichkeit entnahm den Äußerungen, dass die
Klinik die Situation nicht ernst nimmt und darüber hinaus nicht unter Kontrolle hat. Dies schlägt sich natürlich auf das Image der Klinik nieder, das Vertrauen in die sensible Einrichtung 'Krankenhaus' geht verloren.

\section{Kontinuierlicher Kontakt mit den Stakeholdern}

Um für eine Krisensituation gewappnet zu sein, müssen auch die relevanten Ansprechpartner und Anspruchsgruppen bekannt sein. Diese reichen von den Mitarbeitern des Klinikums über das Gesundheitsamt, das Robert-Koch-Institut (RKI), die Deutsche Gesellschaft für Krankenhaushygiene, den Bürgermeister und die Patientenverbände bis hin zur Verbraucherzentrale. Denn in diesen Gruppen finden sich im Falle eines Falles Verbündete, Freunde oder auch Gegner des Klinikums. Durch einen regelmäßigen Kontakt werden wichtige Allianzen geschmiedet und Verbündete für Krisenzeiten gefunden.

\section{Fazit: Die Krise an der Wurzel packen!}

Krankenhäuser sind besonders sensible Institutionen, in denen bereits ein kleiner Fehler ernste Konsequenzen haben kann. Reibungslose Abläufe im Klinikalltag sind daher die beste Krisenprophylaxe. Dies betrifft sowohl die klinischen Prozesse als auch die Kommunikationsroutine. An diesen zentralen Punkten muss die Krisenprävention ansetzen: Interne Abläufe müssen optimiert werden (Risikoanalyse, Status Quo), um das Risikopotenzial zu minimieren. Dabei müssen auch die Anforderungen der externen Kommunikation berücksichtigt werden, damit der Dialog mit der Öffentlichkeit auch in einer Extremsituation „rund“ läuft. All dies kann in eigener Regie funktionieren. Manchmal ist es aber hilfreich, sich hier Hilfe zu suchen, denn ein außenstehender, nicht in die Klinikstrukturen integrierter Berater hat oft einen klareren Blick auf interne Strukturen.

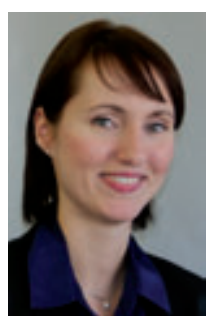

Korrespondenz Rahel Huhn wbpr Public Relations $\mathrm{GmbH}$ Münchner Straße 18 85774 Unterföhring rahel.huhn@wbpr.de 